\title{
Targeted therapies with companion diagnostics in the management of breast cancer: current perspectives
}

This article was published in the following Dove Press journal:

Pharmacogenomics and Personalized Medicine

22 January 2016

Number of times this article has been viewed

\section{Meagan B Myers}

Division of Genetic and Molecular Toxicology, National Center for Toxicological Research, US Food and Drug Administration, Jefferson, AR, USA
Correspondence: Meagan B Myers Division of Genetic and Molecular Toxicology, National Center for Toxicological Research, US Food and Drug Administration, 3900 NCTR

Rd, Jefferson, AR 72079, USA

Tel +l 8705437574

Fax +l 8705437393

Email meagan.myers@fda.hhs.gov

\begin{abstract}
Breast cancer is a multifaceted disease exhibiting both intertumoral and intratumoral heterogeneity as well as variable disease course. Over 2 decades of research has advanced the understanding of the molecular substructure of breast cancer, directing the development of new therapeutic strategies against these actionable targets. In vitro diagnostics, and specifically companion diagnostics, have been integral in the successful development and implementation of these targeted therapies, such as those directed against the human epidermal growth factor receptor 2. Lately, there has been a surge in the development, commercialization, and marketing of diagnostic assays to assist in breast cancer patient care. More recently, multigene signature assays, such as Oncotype DX, MammaPrint, and Prosigna, have been integrated in the clinical setting in order to tailor decisions on adjuvant endocrine and chemotherapy treatment. This review provides an overview of the current state of breast cancer management and the use of companion diagnostics to direct personalized approaches in the treatment of breast cancer.
\end{abstract}

Keywords: HER2, precision medicine, in vitro diagnostics, estrogen receptor, multigene assay

\section{Introduction}

Breast cancer is the second leading cause of cancer mortality in women in the USA, with $\sim 40,000$ deaths per year. ${ }^{1}$ The American Cancer Society estimates 232,000 new cases of invasive breast cancer and $\sim 60,000$ cases of ductal carcinoma in situ will occur this year. ${ }^{1}$ Advances in molecular diagnostics have revealed that breast cancer is not a single disease entity; rather, it is a diverse disease with extensive intertumoral and intratumoral heterogeneity (ie, subclones of cells with differing genetic, epigenetic, and/or phenotypic characteristics). This heterogeneity has significant clinical and therapeutic consequences in terms of patient prognosis and response to hormonal and targeted therapies, in addition to response to chemotherapies.

Growing knowledge of the molecular underpinnings comprising the etiology of cancer has driven the field of personalized or "precision" medicine to identify specific tumor characteristics and exploit these features by developing targeted therapies against these entities. The ability to predict an individual's response to a specific therapy is the ultimate goal in modern precision medicine. Several targeted cancer therapies are currently utilized in standard oncological care as a result of the more detailed genetic and clinical understanding of individual tumor characteristics. The therapeutic use of molecular biomarkers with predictive clinical and pharmacological relevance relies on accurately detecting and/or quantifying these biomarkers to direct the safe and effective treatment of targeted therapies. As a result, the concept of drug-diagnostic codevelopment, or "companion diagnostics", has emerged and is now the foundation of 
personalized cancer medicine. According to the US Food and Drug Administration (FDA) guidance document for industry and FDA staff, "In-Vitro Companion Diagnostic Devices", a companion diagnostic is defined as "an in vitro diagnostic device that provides information that is essential for the safe and effective use of a corresponding therapeutic product". ${ }^{2}$ In addition, the FDA provides four criteria specifying how a companion diagnostic could be essential for the safe and effective use of its corresponding therapeutic. These criteria are to: 1) identify those who would benefit from a therapeutic product, 2) identify those who are at increased risk of serious adverse reactions as a result of treatment with a therapeutic product, 3) identify patients for whom the therapeutic product has been adequately studied and found safe and effective, and 4) monitor response to a therapeutic product for the purpose of adjusting dose or treatment. The FDA guidance document further stipulates that the use of a companion diagnostic with a therapeutic product must be included in the labeling instructions for both the therapeutic product and corresponding diagnostic test.

A molecular diagnostic tool was first used to predict response to a breast cancer therapy in the 1970s, when a high degree of correlation between the presence of the estrogen receptor and a positive treatment response was observed after treatment with the selective estrogen receptor modulator, tamoxifen. ${ }^{3}$ Subsequent clinical trials confirmed these observations, ${ }^{4-6}$ and tamoxifen is now the standard of care for both pre- and postmenopausal women with early or advanced hormone receptor-positive breast tumors. In 1998, the codevelopment and FDA approval of the monoclonal antibody trastuzumab (Herceptin, Roche/Genentech) and the immunohistochemistry (IHC) assay HercepTest (Dako Denmark A/S, Glostrup, Denmark) for the detection of human epidermal growth factor receptor 2 (HER2) protein overexpression in breast tumors demonstrated the value of the drug-diagnostic codevelopment model, spurring avenues of pharmaceutical and biotechnical collaboration and ushering a new era of personalized medicine.

\section{Overview of breast cancer management}

Surgery is the first course of action for operable tumors after a positive pathological diagnosis obtained via a core or fine needle biopsy. For early-stage breast cancer, surgery consists of resection of the primary tumor (lumpectomy) with the aim of negative tumor margins to reduce the risk of local recurrence, and no axillary lymph node dissection is performed if sentinel biopsies of the axillary lymph nodes are negative. ${ }^{7}$ For more advanced breast cancer, either locally invasive or metastatic, surgery options include lumpectomy or total mastectomy. Neoadjuvant therapy has been successfully given to shrink large, invasive tumors to facilitate the process of breast conservation surgery. Recent clinical trials suggest if sentinel lymph nodes are positive, axillary lymph node dissection does not impart an additional survival benefit over lumpectomy plus whole breast irradiation ${ }^{8}$ or lumpectomy plus irradiation and/or chemotherapy. ${ }^{9}$ However, caution against automatically dismissing lymph node dissection is advised, and an individualized approach based on axillary tumor burden and the molecular etiology of the cancer should be considered..$^{10}$ Radiological treatment is indicated after breast-conserving surgery in both early and advanced breast cancers, and after mastectomy, if high-risk factors of recurrence are present (eg, $\geq 5 \mathrm{~cm}$ tumor, lymph node involvement, positive tumor margins).

Multiple clinical and pathological features used in the management of breast cancer are predictive indicators of both patient prognosis and likely therapeutic benefit of various treatment options. The standard descriptors such as patient age, tumor size, axillary lymph node status, histological features (such as tumor grade and lymphovascular infiltration), estrogen receptor and progesterone receptor status, and HER2 overexpression are collectively considered when constructing breast cancer indices and criteria. ${ }^{11-14}$ Additionally, the increasingly popular online webbased tools such as Adjuvant! (http://www.adjuvantonline. $\underline{\text { com) }}$ ) and PREDICT (http://www.predict.nhs.uk) are used for patient prognosis. Modern molecular profiling has subsequently categorized breast cancer into primarily four intrinsic subtypes, luminal A, luminal B, HER2-enriched, and basal-like, which differ in their patterns of gene expression, clinical features, prognosis, and response to treatment. ${ }^{15,16}$ Clinicopathological surrogate definitions of the intrinsic subtypes include luminal A-like, luminal B-like, HER2-positive, and triple-negative. Luminal A-like tumors are hormone receptor-positive (estrogen receptor-positive/ progesterone receptor-positive) and HER2-negative. Luminal B-like tumors are characterized as hormone receptor-positive (estrogen receptor-positive/progesterone receptor-positive/ negative) and HER2 overexpressed/amplified, or hormone receptor-positive (estrogen receptor-positive/progesterone receptor-negative) and HER2 negative. In HER2-enriched tumors, HER2 is overexpressed/amplified and hormone receptors are absent. For triple-negative tumors, hormone receptors and HER2 are absent. ${ }^{17}$ Luminal A tumors are the most common and have relatively low proliferation potential 
and good prognosis. ${ }^{16,18,19}$ Luminal B tumors are characterized as highly proliferative and have somewhat worse prognosis than luminal A. ${ }^{16,18,19}$ Both the HER2 and basal-like subtypes are highly proliferative and confer poor prognoses. Differences in sensitivity to therapeutic treatments are also a function of their molecular signatures. Luminal A tumors are highly sensitive to hormone therapy, due to increased expression of hormone receptors, yet are poorly sensitive to chemotherapy. Luminal B tumors, however, are considered to have incomplete sensitivity to hormone therapy, yet derive some benefits from chemotherapy. ${ }^{20,21}$ HER2-enriched and basal-like subtypes are insensitive to hormone therapy but are sensitive to chemotherapy. ${ }^{20}$ Sensitivity to biologics targeted against the HER2 receptor, such as trastuzumab, pertuzumab, ado-trastuzumab emtansine, and lapatinib, is a function of the overexpression/amplification of the HER2 gene in luminal B/HER2-positive and HER2-enriched tumors.

Adjuvant systemic therapy is standard care after surgery in pre- and postmenopausal women. For early-stage breast cancer, adjuvant therapy is used to eradicate possible micrometastatic tumors in an attempt to prevent recurrence and offer a cure. For advanced, metastatic cancer, the goal of chemotherapy is to control disease burden and improve survival. Approximately $80 \%$ of breast cancers are hormone receptor-positive and for these patients, endocrine therapies, in the form of selective estrogen receptor modulators or aromatase inhibitors, are preferred over chemotherapy as a first-line treatment. Recent American Society of Clinical Oncology (ASCO) guidelines ${ }^{22}$ recommend that tamoxifen therapy should be given to premenopausal women for a total of 10 years based on clinical trials demonstrating reduced recurrence and improved survival benefit with longer duration of tamoxifen use. ${ }^{23,24}$ For postmenopausal women, 10 years total endocrine therapy is recommended, which consists of either tamoxifen given for the full duration or 5 years tamoxifen plus 5 years of an aromatase inhibitor. ${ }^{22}$ For tumors that overexpress HER2, trastuzumab-based chemotherapy is now the standard of care and it has improved the rate of response, time to progression, and the overall survival in patients with metastatic breast cancer, as well as diseasefree and overall survival in patients with localized invasive breast cancer. ${ }^{25-29}$ Patients also benefit from three other HER2 targeted therapies, pertuzumab, ado-trastuzumab emtansine, and lapatinib, which have been approved for use for treatment of HER2-positive breast cancer. ${ }^{30-32}$ Triple-negative breast cancer, which lacks the expression of the estrogen receptor, progesterone receptor, and HER2, is an aggressive subgroup for which no targeted therapies are available; therefore, systemic chemotherapy is the only option for these patients.

\section{Companion diagnostics in breast cancer}

All FDA-approved companion diagnostics were utilized in breast cancer test for the presence of HER2 overexpression either at the protein level or overamplification of the HER2 gene. Given the history of their use, estrogen receptor and progesterone receptor testing is not considered companion diagnostics by the FDA; however, determination of the presence of these tumor markers is mandatory for the effective treatment with aromatase inhibitors and the hormone modulators, tamoxifen and fulvestrant. Therefore, estrogen receptor and progesterone receptor testing is included in the discussion below.

\section{Estrogen receptor and progesterone receptor testing}

In normal breast tissue, estrogen and progesterone are critical regulators of the growth and differentiation of epithelial cells. Specifically, estrogen and progesterone mediate their biological effects through binding to isoforms of their respective steroid receptors, the estrogen receptor and progesterone receptor. The estrogen receptor- $\alpha$ isoform is the most studied and abundant hormone receptor in breast, with upward of $70 \%$ of tumors expressing this receptor. ${ }^{33}$ High levels of estrogen and/or prolonged exposure to estrogen promote breast carcinogenesis, likely through increased signaling and upregulation of estrogen responsive genes that trigger DNA synthesis and cellular proliferation. Endocrine therapies, such as tamoxifen, which is an estrogen receptor antagonist in the breast, or aromatase inhibitors, which prevent the conversion of androgens to estrogen, block estrogen signaling and estrogen-driven cellular proliferation in breast tissue.

IHC is the primary testing method for assessing the presence of the estrogen and progesterone receptors in breast cancer. First standardized and validated by Harvey et al, ${ }^{34} \mathrm{IHC}$ is routinely performed on resected breast tumor specimens or core needle biopsies. However, the use of both technically and clinically unvalidated IHC assays has resulted in widespread inter-laboratory variability. Multiple components of IHC variability exist, including preanalytic sampling variability (eg, source of tumor sample [core needle or resected tumor], differences in fixation and preparation methodologies), analytic variability (eg, sensitivity of the assay, type of reagents and antibody used, staining time, and use of proper controls), and postanalytic variability (eg, method of scoring and established 
cutoff value defining positivity). ${ }^{35}$ In 2010, the ASCO/College of American Pathologists (CAP) guideline recommendations were published to minimize inter-laboratory variability. ${ }^{36}$ A crucial component of the ASCO/CAP guideline is that estrogen and progesterone receptor assays be considered positive if $1 \%$ or more tumor cells are positive for receptor expression. Previously, $\geq 10 \%$ positivity was required to be considered eligible for hormone therapy. Since patients with as low as $1 \%$ of tumor cells positive for estrogen and progesterone staining derive benefit from endocrine therapy, this recommendation has expanded the patient population eligible for these well-tolerated therapies. The Dako ER/PR pharmDx assay kit (Dako Denmark A/S) was FDA 510(k)cleared in 2005 and is the only in vitro diagnostic kit currently approved by the ASCO. ${ }^{36}$

\section{HER2 and corresponding companion diagnostics}

HER2 (gene designation: ERRB2, HER2/neu), encoded by the protooncogene $H E R 2 / n e u$ on chromosome 17 , is a member of the epidermal growth factor receptor family of type I transmembrane receptor tyrosine kinases (consisting of four members: HER1, HER2, HER3, and HER4). Through a plethora of intracellular signaling pathways, HER-family receptors mediate normal cell growth, motility, differentiation, and survival. ${ }^{37}$ HER2 is unique among the HER-family of receptors, as it is an orphan receptor (ie, has no known naturally occurring ligand), remains in a fixed open conformation (resembling a ligand-activated state), and is the preferred partner for heterodimerization with HER-family receptors, resulting in increased signaling potency as compared with dimerization with other members of the HER-family. ${ }^{38}$ First reported by Slamon et $\mathrm{al}^{39}$ in 1987, HER2 overexpression was shown to be present in $20 \%-30 \%$ of breast cancers and resulted in shorter time to relapse and reduced overall survival. In breast cancer, HER2 receptor overexpression results primarily from amplification of the HER2 gene; ${ }^{40}$ however, mutations in the HER2 gene $^{41}$ and transcriptional and posttranscriptional alterations may be mechanisms involved as well. ${ }^{42}$ This, in turn, increases HER2 receptor density (up to $\sim 2$ million) on the cellular surface, and results in excessive HER2 receptor homodimerization or HER-family heterodimerization (ie, HER2:HER3) in a ligand-dependent manner. ${ }^{43}$ Rampant downstream intracellular signaling follows, occurring through multiple pathways, including the PI3K/AKT/mTOR, ERK, JAK/STAT, Ras/Raf, Rho/Rac, and phospholipase $\mathrm{C} \gamma$ pathways, which drive cellular proliferation, invasion, and survival. ${ }^{38}$ Aberrant levels of HER2 in breast cancer correlate with an aggressive phenotype and poor prognosis, including an increased risk of metastases and recurrence, poorer relapse-free survival, and shorter overall survival. ${ }^{39,44}$ This poorer outcome extends to those patients with small $(<1 \mathrm{~cm})$, node-negative, HER2-positive breast cancer, which was shown to have 2.68 times greater risk of recurrence and 5.3 times greater risk of metastasis, as compared to those patients with HER2-negative tumors. ${ }^{45}$

HER2 overexpression and the presence of abundant, accessible HER2 protein on the cellular surface in 20\%-25\% of breast cancers render HER2 an ideal actionable molecule for targeted therapy. Trastuzumab was the first FDA-approved biologic for the treatment of HER2-positive breast cancer and has been shown to significantly improve survival in the neoadjuvant, adjuvant, and metastatic settings. ${ }^{25-29,46}$ Trastuzumab is a recombinant, humanized monoclonal antibody which recognizes and binds to the extracellular domain of HER2, preventing receptor dimerization. Trastuzumab inhibits growth and induces cancer cell death by multiple mechanisms of action, including: 1) activation of antibody-dependent cellular toxicity, 2) blockage of the proteolytic cleavage of the extracellular domain, 3) inhibition of intracellular signal transduction, 4) inhibition of tumor-induced angiogenesis, and 5) inhibition of cancer treatment-induced DNA damage repair. ${ }^{47-49}$ Enrollment for the pivotal Phase III clinical trial began in 1995, utilizing an enriched clinical trial design where only HER2-positive patients (as determined by an IHC clinical trial assay) were included. ${ }^{29}$ Shortly after initiation, Genetech, the manufacturer of trastuzumab, partnered with the diagnostic company Dako Denmark A/S to develop a commercial test to identify the overexpression of HER2 in breast cancer samples. The results of the Phase III clinical trial not only demonstrated the superiority of first-line trastuzumabbased chemotherapy compared to chemotherapy alone, but also validated the approach of an enriched clinical trial design based on results of diagnostic testing for a specific molecular target, in this case HER2. In 1998, trastuzumab and the Dako Denmark A/S companion diagnostic test, HercepTest, received simultaneous FDA approval for use in women with HER2-positive metastatic breast cancer.

Due to the high prognostic and predictive value of HER2, HER2 testing (preferentially by an FDA-approved IHC, bright-field in situ hybridization, or fluorescent in situ hybridization [FISH] assay) is now recommended for all primary, metastatic, and recurrent breast cancers. ${ }^{44,50}$ Currently, there are seven active FDA-approved in vitro companion diagnostic devices to detect HER2 overexpression in breast cancer (three with premarket approvals withdrawn) (Table 1). Of the 
active diagnostics, three are IHC assays for the staining and visualization of the HER2 protein. These are: 1) HercepTest (Dako Denmark A/S), 2) PATHWAY anti-HER2/new (4B5) rabbit monoclonal antibody (Ventana Medical Systems, Inc., Tucson, AZ, USA), and 3) Bond Oracle HER2 IHC system (Leica Biosystems, Wetzlar, Germany). The remaining four tests quantify HER 2 gene copy number; two via FISH assays (PathVysion HER2 DNA probe kit [Abbott Laboratories, Abbott Park, IL, USA] and HER2 IQFISH pharmDx kit [Dako Denmark A/S]), one by chromogenic in situ hybridization (CISH) assay (HER2 CISH pharmDx kit [Dako Denmark $\mathrm{A} / \mathrm{S}]$ ), and one by dual in situ hybridization assay (INFORM HER2 Dual ISH DNA probe cocktail [Ventana Medical Systems, Inc.]). All are FDA-approved for use as a companion diagnostic to assess suitability for initiation of trastuzumab treatment. HercepTest is the only companion diagnostic approved for use in treatment with trastuzumab, pertuzumab, and ado-trastuzumab.

IHC has been the standard testing method for the evaluation of HER2 positivity due to its ease of use, availability, use of routine microscopy, and the ability to archive stained slides. IHC testing utilizes specific monoclonal or polyclonal antibodies which bind to the HER2 protein on the cellular surface. The addition of a secondary biotinylated antibody, followed by an enzymatic reaction, yields a colorimetric intensity that is proportional to the amount of HER2 protein present. For the FDA-approved HER2 IHC companion diagnostics, different primary antibodies are used: A085 (polyclonal; HercepTest), 4B5 (rabbit monoclonal; PATHWAY), and CB11 (mouse monoclonal; Bond Oracle). Unlike the qualitative IHC assessment for the presence of the estrogen receptor and progesterone receptor, IHC testing for overexpression of HER2 is considered quantitative because HER2 is expressed in all breast epithelial cells. As with IHC testing of the estrogen receptor and progesterone receptor, variability in HER2 IHC may arise from preanalytical, analytical, and postanalytical factors. To reduce inter-laboratory variability, recommendations on the grading and scoring of HER2 IHC were published by the ASCO/CAP and are based on

Table I List of FDA-approved (active and withdrawn) companion diagnostics for use in HER-positive breast cancer ${ }^{a}$

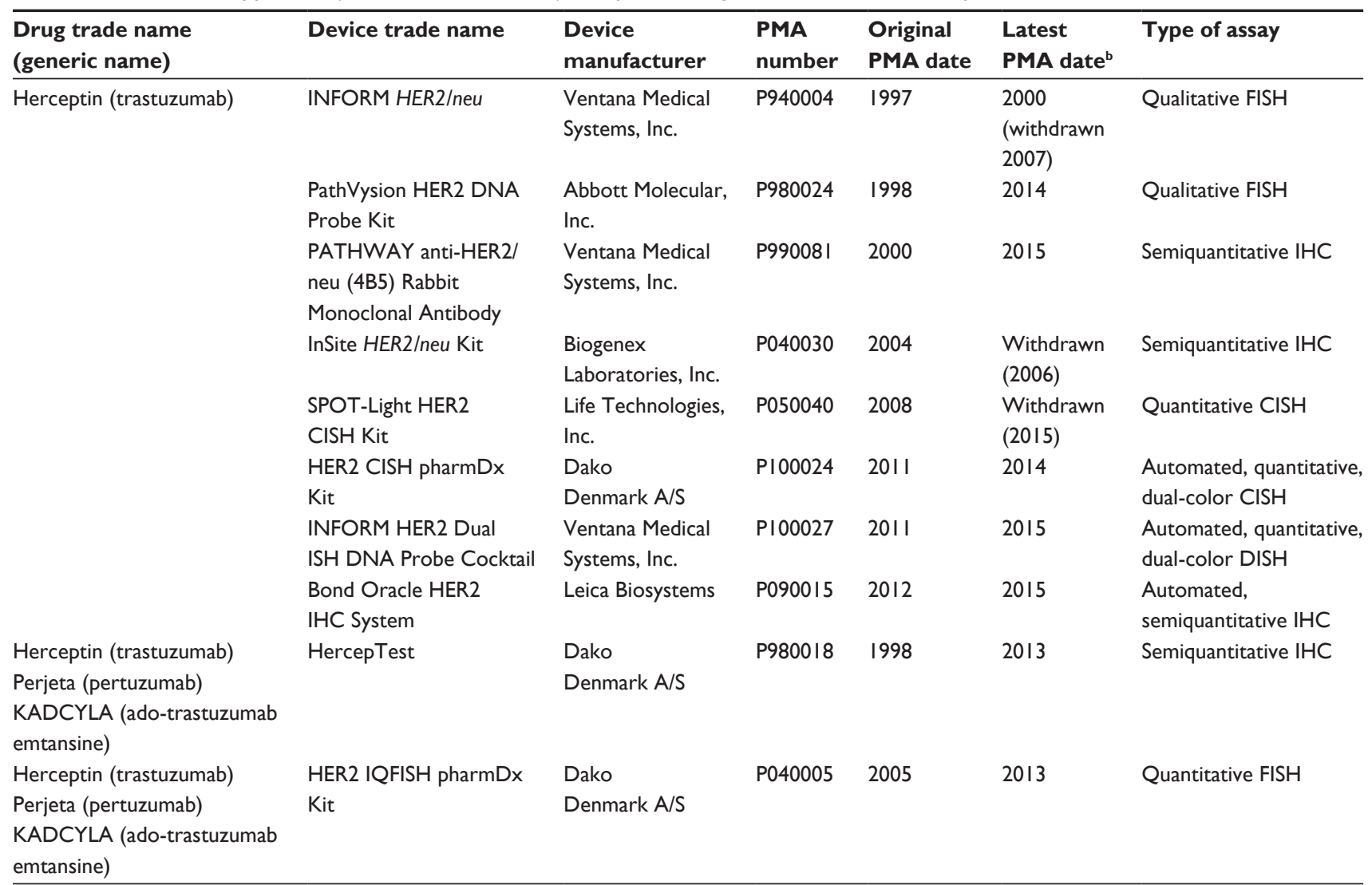

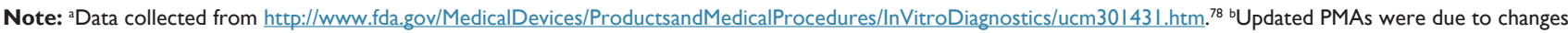
in manufacturing, product specifications, addition of drugs in the labeling, software, etc.

Abbreviations: $\mathrm{CISH}$, chromogenic in situ hybridization; DISH, dual in situ hybridization; FDA, US Food and Drug Administration; FISH, fluorescent in situ hybridization; HER2, human epidermal growth factor receptor 2; IHC, immunohistochemistry; PMA, premarket approval. 
a $0,1+, 2+$, and $3+$ tiered scoring system: 0 , no staining observed or incomplete, faint/barely perceptible membrane staining in $\leq 10 \%$ of tumor cells; $1+$, incomplete membrane staining that is faint/barely perceptible and within $>10 \%$ of tumor cells; $2+$, incomplete and/or weak/moderate circumferential membrane staining in $\leq 10 \%$ of tumor cells; and $3+$, complete and intense circumferential staining within $>10 \%$ of tumor cells. ${ }^{44,50}$ The scores 0 and $1+$ are considered negative; $2+$, equivocal; and $3+$ positive. For those samples with test results that are equivocal, additional testing with an alternative method, such as FISH, is recommended.

FISH, CISH, and silver-enhanced in situ hybridization assays quantify the HER2 gene copy number per cell using a single- or dual-probe technique. FISH has become a widely accepted platform for HER2 testing, producing equivocal results in less than $5 \%$ of cases. FISH is considered more accurate, reproducible, and robust than IHC and is less affected by preanalytic and analytic variables. However, these assays are costly, labor-intensive, and require fluorescent microscopy and advanced training. Also because they are subject to photobleaching, archival storage is intractable. Next-generation bright-field in situ hybridization assays such as CISH and silverenhanced in situ hybridization eliminate the need for fluorescent microscopy, cost less, and are more stable over time. Newly automated systems such as INFORM HER2 Dual ISH, which combines silver-enhanced in situ hybridization for HER2 copy number and CISH for enumeration of chromosome 17, are gaining favorability due to the reduced variability and labor associated with this system.

All FDA-approved in situ hybridization-based HER2 companion diagnostics employ a dual-probe technique on paraffin-embedded tumor samples. These dual-probe assays use fluorescent-labeled (eg, FISH) or chromogenic-labeled (eg, CISH) DNA probes to compare the abundance of HER2 gene copies to that of the number of chromosome 17 centromeres, as determined by the centromere enumeration probe 17 (CEP17), per interphase cell. The 2013 ASCO/ CAP guidelines consider a sample to be HER2 positive if the HER2/CEP17 ratio is $\geq 2.0$ or the HER2 copy number is $\geq 6.0$ signals/cell. ${ }^{44,50} \mathrm{~A}$ sample is considered negative if the HER2/CEP17 ratio is $<2.0$ and the average HER 2 copy number is $<4.0$ signals/cell. For equivocal cases, where the HER2/CEP17 ratio is $<2.0$ but the average HER 2 copy number is $\geq 4.0$ and $<6.0$ signals/cell, reflex testing on the same specimen (by IHC or FISH with an alternative chromosome
17 probe) or testing on a new section of the specimen (FISH or IHC) should be performed.

\section{Multigene signatures in prognosis and prediction to adjuvant therapy}

Over the last 2 decades, remarkable improvements have been made in the molecular diagnostics and therapeutic interventions utilized in breast cancer patient care. However, challenges do exist, particularly in stratifying which operable patients are at the greatest risk of recurrence and, therefore, would benefit from systemic cytotoxic chemotherapy. Traditionally, the decision to treat with systemic adjuvant chemotherapy was based on clinicopathological factors, such as patient age, tumor type, size, and grade, and axillary lymph node involvement, as well as patient and physician preferences. However, this approach results in exposing a large fraction of breast cancer patients to potentially lifethreatening chemotherapy-associated toxicities who would otherwise gain little or no improvement in clinical outcome. ${ }^{51}$ Advancements in understanding the molecular signaling pathways and genetic signatures of breast cancer have progressed the field forward resulting in new methodologies to aid in the decision of which patients should and should not receive systemic adjuvant chemotherapy. The development and commercialization of multigene expression-based assays, such as Oncotype DX, MammaPrint, and Prosigna, have resulted in a paradigm shift in the management and treatment of breast cancer, specifically in the care of early-stage breast cancer. Although these assays are not companion diagnostics per se (as they are not linked to a particular drug), changes in clinical decisions and treatment course do occur based on outcome predictions from these assays, and thus are included and discussed below.

Oncotype DX (Genomic Health, Redwood City, CA, USA) is a quantitative real-time reverse transcriptase polymerase chain reaction assay widely used in the USA. This assay quantifies the gene expression level of 21 genes, including 16 cancer-related genes and five reference genes. ${ }^{52,53}$ The cancer genes assessed in this panel are primarily associated with proliferation (ie, Ki67 and CCNB1), estrogen regulation (ie, estrogen receptor and progesterone receptor), HER2 amplification, and invasiveness. This assay is performed on RNA extracted from formalin-fixed paraffin-embedded primary breast tumor samples in a Clinical Laboratory Improvement Amendments-regulated central laboratory. The Oncotype DX assay utilizes an algorithm, based on the expression profile of the 16 cancer-related genes, to generate a recurrence score (RS) which ranks a patient's 10-year risk 
of recurrence as low $(\mathrm{RS}<18)$, intermediate ( RS 18-30), or high ( $\mathrm{RS} \geq 31)$. Originally validated in estrogen receptorpositive, node-negative, tamoxifen-treated patients, ${ }^{53}$ the Oncotype DX RS has been shown to accurately predict patients with a low RS to have little to no benefit from chemotherapy due to their low risk of recurrence. Whereas, patients with a high RS predicted in the Oncotype DX test are likely to experience breast cancer recurrence within 10 years and thus would benefit from a more aggressive treatment course ${ }^{54}$ Oncotype DX has been validated in multiple clinical trials, including both node-negative and node-positive, estrogen receptor-positive patients. ${ }^{54-58}$ Most recently, the initial results of the large, prospectively conducted, randomized clinical trial, the Trial Assigning Individualized Options for Treatment, were published. Sparano et al ${ }^{59}$ reported that estrogen receptor-positive, node-negative, HER2-negative patients with a low Oncotype DX RS $(\leq 10)$ receiving endocrine therapy alone $(n=1,626)$ resulted in a 5 -year invasive disease-free survival and distant recurrence-free survival rates of $93.8 \%$ and $99.3 \%$, respectively. Although all patients met the National Comprehensive Cancer Network guidelines for recommendation of adjuvant chemotherapy, the overall 5 -year survival was $98.0 \%$, demonstrating endocrine therapy alone may be sufficient for the vast majority of low $(\leq 10)$ RS patients. Results for the clinical utility of the Oncotype DX to predict benefit of adjuvant chemotherapy in patients with intermediate RSs (11-25) are forthcoming. Considering these trial results, Oncotype DX is presently the only multigene expression-based assay with Level I evidence both as a prognostic tool as well as predicting response to adjuvant therapy in estrogen receptor-positive, node-negative patients. ${ }^{60}$ Further, Level II evidence was reached for validation as a prognostic tool in estrogen receptor-positive, nodepositive patients. The evidence prompted both the National Comprehensive Cancer Network ${ }^{61}$ and $\mathrm{ASCO}^{62}$ to include Oncotype DX RS analysis in their recommendations for testing estrogen receptor-positive, HER2-negative, node-negative breast cancer patients.

Oncotype DX is currently marketed as a laboratory developed test and has not undergone FDA approval (declared exempt from FDA premarket clearance). According to the FDA, a laboratory developed test is "an in vitro diagnostic device that is intended for clinical use and designed, manufactured and used within a single laboratory". ${ }^{63}$ Oncotype DX, as well as MammaPrint and Prosigna, is further categorized as a particular subset of laboratory developed tests, termed in vitro diagnostic multivariate index assays. These assays are defined as a device that:
1) combines the values of multiple variables using an interpretation function to yield a single, patient-specific result (eg, a "classification", "score", "index", etc), that is intended for use in the diagnosis of disease or other conditions, or in the cure, mitigation, treatment or prevention of disease, and 2) provides a result whose derivation is nontransparent and cannot be independently derived or verified by the end user. ${ }^{64}$

Historically, the FDA did not require premarket clearance and approval of laboratory developed tests if the test was performed under the Clinical Laboratory Improvement Amendments regulations and was not marketed for distribution. However, as the complexity and clinical impact of these assays has grown (eg, in vitro diagnostic multivariate index assays), so too has the risk of misdiagnosis and mistreatment. In 2014, the FDA released the draft guidance document "Framework for Regulatory Oversight of Laboratory Developed Tests" ${ }^{\prime 63}$ which describes the agency's proposed risk-based oversight for laboratory developed tests, including premarket review of higher risk laboratory tests, such as those with the same intended use as a FDA-cleared or -approved companion diagnostic test (ie, direct patient therapy decisions).

In 2007, the prognostic MammaPrint 70-Gene Breast Cancer Recurrence Assay (Agendia, Irvine, CA, USA and Amsterdam, the Netherlands) became the first multigene expression assay to gain FDA approval through the 510(k) clearance process as a Class II in vitro diagnostic multivariate index assay. This assay utilizes DNA microarray technology to assess the expression of 70 genes involved in multiple cellular and cancer-associated processes such as cell cycle, proliferation, invasion, angiogenesis, and metastasis. MammaPrint stratifies patients into binary low- or high-risk prognostic classifications for prediction of distant recurrence based on gene expression profiling reported by van't Veer et al. ${ }^{65}$ The study by van't Veer et al compared a 70 -gene expression profile in untreated, node-negative, patients who developed metastasis within 5 years to patients who did not. MammaPrint has been validated as a prognostic tool to predict recurrence in both retrospective ${ }^{66,67}$ and prospective studies $^{68,69}$ in node-negative patients, as well as retrospectively in node-positive patients. ${ }^{66,70}$ MammaPrint is FDA-cleared for use in lymph node-negative, estrogen receptor-positive and estrogen receptor-negative fresh frozen and formalin-fixed paraffin-embedded breast tumors that are $\leq 5.0 \mathrm{~cm}$.

Originally proposed by Parker et al, ${ }^{71}$ the Prediction Analysis of Microarrays (PAM50) is a 50-gene expression panel which distinguishes the four intrinsic subtypes of breast cancer 
(luminal A, luminal B, HER2-enriched, and basal-like). The concept behind PAM50 is based on the inherent differences in the expression of key genes, such as ESRl (estrogen receptor gene), $E R R B 2$, and genes involved in cell cycle activation and proliferation, which are known to significantly affect the risk of recurrence. PAM50 generates an individualized risk of RS that is prognostic and predictive of disease recurrence in both untreated and tamoxifen-treated cohorts. ${ }^{71,72}$ Most recently, NanoString Technologies (Seattle, WA, USA) received FDA $510(\mathrm{k})$ clearance to market and distribute the Prosigna Breast Cancer Prognostic Gene Signature Assay. For the Prosigna in vitro diagnostic, NanoString Technologies has adapted the PAM50 with the NanoString nCounter DX Analysis System, which uses multiplexed, gene-specific fluorescently labeled nucleic acid probes. A proprietary algorithm using the data generated from the PAM50 signature in addition to tumor size and nodal status generates a Prosigna risk of RS. The Prosigna risk of RS is stratified as low-, intermediate-, or high-risk for node-negative patients, whereas node-positive patients are stratified as only low- or high-risk category. Multiple validation studies have confirmed this methodology to predict breast cancer recurrence in estrogen receptor-positive, postmenopausal breast cancer patients receiving endocrine therapy. ${ }^{73-76}$ Prosigna is approved for use in hormone receptor-positive, node-negative (stage I or II) or node-positive (stage II) postmenopausal women to be treated with adjuvant endocrine therapy. Recent validation of the Prosigna analytical performance across multiple clinical laboratories supports its use as a decentralized test for breast cancer risk stratification. ${ }^{77}$

\section{Conclusion}

Over the last 2 decades, great advances have been made in the management and treatment of breast cancer. Not only have new drugs and biologics become available but also the overall model for managing and treating breast cancer patients has changed. A "one size fits all" approach is in the past and the era of precision medicine in breast cancer oncology is upon us. Use of tailored approaches will inevitably decrease the number of patients who undergo potentially life-threatening systemic cytotoxic chemotherapy with no realistic expectation of benefit. Additional advances in early detection as well as discovery of new molecular markers and the development of drugs or biologics targeting these specific markers/pathways will advance the field even further.

\section{Acknowledgments}

The author would like to thank Dayton Petibone and Todd Townsend for their critical review of this manuscript. The information in these materials is not a formal dissemination of information by the FDA and does not represent the agency position or policy.

\section{Disclosure}

The author reports no conflicts of interest in this work.

\section{References}

1. Breast Cancer. 2015. Available from: http://www.cancer.org/cancer/ breastcancer/detailedguide/breast-cancer-key-statistics. Accessed May 11, 2015.

2. US Food and Drug Administration. In Vitro Companion Diagnostic Devices. US Food and Drug Administration; 2014.

3. Lerner HJ, Band PR, Israel L, Leung BS. Phase II study of tamoxifen: report of 74 patients with stage IV breast cancer. Cancer Treat Rep. 1976;60(10):1431-1435.

4. Fisher B, Costantino J, Redmond C, et al. A randomized clinical trial evaluating tamoxifen in the treatment of patients with node-negative breast cancer who have estrogen-receptor-positive tumors. $N$ Engl $J$ Med. 1989;320(8):479-484.

5. Fisher B, Redmond C, Brown A, et al. Influence of tumor estrogen and progesterone receptor levels on the response to tamoxifen and chemotherapy in primary breast cancer. J Clin Oncol. 1983;1(4): 227-241.

6. Fisher B, Redmond C, Brown A, et al. Treatment of primary breast cancer with chemotherapy and tamoxifen. N Engl J Med. 1981;305(1): $1-6$.

7. Lyman GH, Temin S, Edge SB, et al. Sentinel lymph node biopsy for patients with early-stage breast cancer: American Society of Clinical Oncology clinical practice guideline update. J Clin Oncol. 2014; 32(13):1365-1383.

8. Caudle AS, Hunt KK, Tucker SL, et al. American College of Surgeons Oncology Group (ACOSOG) Z0011: impact on surgeon practice patterns. Ann Surg Oncol. 2012;19(10):3144-3151.

9. Galimberti V, Cole BF, Zurrida S, et al. Axillary dissection versus no axillary dissection in patients with sentinel-node micrometastases (IBCSG 23-01): a phase 3 randomised controlled trial. Lancet Oncol. 2013;14(4):297-305.

10. Voutsadakis IA, Spadafora S. Axillary lymph node management in breast cancer with positive sentinel lymph node biopsy. World J Clin Oncol. 2015;6(1):1-6.

11. Coates AS, Winer EP, Goldhirsch A, et al. Tailoring therapies - improving the management of early breast cancer: St Gallen International Expert Consensus on the Primary Therapy of Early Breast Cancer 2015. Ann Oncol. 2015;26(8):1533-1546.

12. Rakha EA, El-Sayed ME, Lee AH, et al. Prognostic significance of Nottingham histologic grade in invasive breast carcinoma. J Clin Oncol. 2008;26(19):3153-3158.

13. Carlson RW, Brown E, Burstein HJ, et al. NCCN Task Force Report: adjuvant therapy for breast cancer. J Natl Compr Canc Netw. 2006;4(1): S1-S26.

14. Van Belle V, Van Calster B, Brouckaert O, et al. Qualitative assessment of the progesterone receptor and HER2 improves the Nottingham Prognostic Index up to 5 years after breast cancer diagnosis. J Clin Oncol. 2010;28(27):4129-4134.

15. Perou CM, Sorlie T, Eisen MB, et al. Molecular portraits of human breast tumours. Nature. 2000;406(6797):747-752.

16. Sorlie T, Perou CM, Tibshirani R, et al. Gene expression patterns of breast carcinomas distinguish tumor subclasses with clinical implications. Proc Natl Acad Sci U S A. 2001;98(19):10869-10874.

17. Goldhirsch A, Winer EP, Coates AS, et al. Personalizing the treatment of women with early breast cancer: highlights of the St Gallen International Expert Consensus on the Primary Therapy of Early Breast Cancer 2013. Ann Oncol. 2013;24(9):2206-2223. 
18. Cheang MC, Chia SK, Voduc D, et al. Ki67 index, HER2 status, and prognosis of patients with luminal B breast cancer. J Natl Cancer Inst. 2009;101(10):736-750.

19. Hu Z, Fan C, Oh DS, et al. The molecular portraits of breast tumors are conserved across microarray platforms. BMC Genomics. 2006;7:96.

20. Rouzier R, Perou CM, Symmans WF, et al. Breast cancer molecular subtypes respond differently to preoperative chemotherapy. Clin Cancer Res. 2005;11(16):5678-5685.

21. Sotiriou C, Neo SY, McShane LM, et al. Breast cancer classification and prognosis based on gene expression profiles from a population-based study. Proc Natl Acad Sci U S A. 2003;100(18):10393-10398.

22. Burstein HJ, Temin S, Anderson H, et al. Adjuvant endocrine therapy for women with hormone receptor-positive breast cancer: American Society of Clinical Oncology clinical practice guideline focused update. J Clin Oncol. 2014;32(21):2255-2269.

23. Davies C, Pan H, Godwin J, et al. Long-term effects of continuing adjuvant tamoxifen to 10 years versus stopping at 5 years after diagnosis of oestrogen receptor-positive breast cancer: ATLAS, a randomised trial. Lancet. 2013;381(9869):805-816.

24. Tormey DC, Gray R, Falkson HC. Postchemotherapy adjuvant tamoxifen therapy beyond five years in patients with lymph node-positive breast cancer. Eastern Cooperative Oncology Group. J Natl Cancer Inst. 1996;88(24):1828-1833.

25. McArthur HL, Mahoney KM, Morris PG, et al. Adjuvant trastuzumab with chemotherapy is effective in women with small, node-negative, HER2-positive breast cancer. Cancer. 2011;117(24):5461-5468.

26. Piccart-Gebhart MJ, Procter M, Leyland-Jones B, et al. Trastuzumab after adjuvant chemotherapy in HER2-positive breast cancer. $N$ Engl J Med. 2005;353(16):1659-1672.

27. Rodrigues MJ, Peron J, Frenel JS, et al. Benefit of adjuvant trastuzumabbased chemotherapy in T1ab node-negative HER2-overexpressing breast carcinomas: a multicenter retrospective series. Ann Oncol. 2013;24(4):916-924.

28. Slamon D, Eiermann W, Robert N, et al. Adjuvant trastuzumab in HER2positive breast cancer. $N$ Engl J Med. 2011;365(14):1273-1283.

29. Slamon DJ, Leyland-Jones B, Shak S, et al. Use of chemotherapy plus a monoclonal antibody against HER2 for metastatic breast cancer that overexpresses HER2. N Engl J Med. 2001;344(11):783-792.

30. Baselga J, Cortes J, Kim SB, et al. Pertuzumab plus trastuzumab plus docetaxel for metastatic breast cancer. $N$ Engl J Med. 2012;366(2): 109-119.

31. Geyer CE, Forster J, Lindquist D, et al. Lapatinib plus capecitabine for HER2-positive advanced breast cancer. $N$ Engl J Med. 2006;355(26): 2733-2743.

32. Verma S, Miles D, Gianni L, et al. Trastuzumab emtansine for HER2-positive advanced breast cancer. $N$ Engl J Med. 2012;367(19): 1783-1791.

33. Clark GM, Osborne CK, McGuire WL. Correlations between estrogen receptor, progesterone receptor, and patient characteristics in human breast cancer. J Clin Oncol. 1984;2(10):1102-1109.

34. Harvey JM, Clark GM, Osborne CK, Allred DC. Estrogen receptor status by immunohistochemistry is superior to the ligand-binding assay for predicting response to adjuvant endocrine therapy in breast cancer. J Clin Oncol. 1999;17(5):1474-1481.

35. Allred DC, Carlson RW, Berry DA, et al. NCCN Task Force Report: Estrogen receptor and progesterone receptor testing in breast cancer by immunohistochemistry. J Natl Compr Canc Netw. 2009;7(6):S1-S21; quiz S22-S23.

36. Hammond ME, Hayes DF, Dowsett M, et al. American Society of Clinical Oncology/College of American Pathologists guideline recommendations for immunohistochemical testing of estrogen and progesterone receptors in breast cancer. J Clin Oncol. 2010;28(16): 2784-2795.

37. Yarden Y, Sliwkowski MX. Untangling the ErbB signalling network. Nat Rev Mol Cell Biol. 2001;2(2):127-137.

38. Rubin I, Yarden Y. The basic biology of HER2. Ann Oncol. 2001;12(1): S3-S8.
39. Slamon DJ, Clark GM, Wong SG, Levin WJ, Ullrich A, McGuire WL. Human breast cancer: correlation of relapse and survival with amplification of the HER-2/neu oncogene. Science. 1987;235(4785): 177-182.

40. Pauletti G, Godolphin W, Press MF, Slamon DJ. Detection and quantitation of HER-2/neu gene amplification in human breast cancer archival material using fluorescence in situ hybridization. Oncogene. 1996;13(1):63-72.

41. Bose R, Kavuri SM, Searleman AC, et al. Activating HER2 mutations in HER2 gene amplification negative breast cancer. Cancer Discov. 2013;3(2):224-237.

42. Nelson EL. HER2/neu: an increasingly important therapeutic target. Part 1: basic biology and therapeutic armamentarium. Clin Investig. 2014;4(7):649-671.

43. Landgraf R. HER2 therapy. HER2 (ERBB2): functional diversity from structurally conserved building blocks. Breast Cancer Res. 2007;9(1):202.

44. Wolff AC, Hammond ME, Hicks DG, et al. Recommendations for human epidermal growth factor receptor 2 testing in breast cancer: American Society of Clinical Oncology/College of American Pathologists clinical practice guideline update. J Clin Oncol. 2013;31(31):3997-4013.

45. Gonzalez-Angulo AM, Litton JK, Broglio KR, et al. High risk of recurrence for patients with breast cancer who have human epidermal growth factor receptor 2-positive, node-negative tumors $1 \mathrm{~cm}$ or smaller. J Clin Oncol. 2009;27(34):5700-5706.

46. Gianni L, Eiermann W, Semiglazov V, et al. Neoadjuvant and adjuvant trastuzumab in patients with HER2-positive locally advanced breast cancer (NOAH): follow-up of a randomised controlled superiority trial with a parallel HER2-negative cohort. Lancet Oncol. 2014;15(6): 640-647.

47. Hudis CA. Trastuzumab - mechanism of action and use in clinical practice. N Engl J Med. 2007;357(1):39-51.

48. Spector NL, Blackwell KL. Understanding the mechanisms behind trastuzumab therapy for human epidermal growth factor receptor 2-positive breast cancer. J Clin Oncol. 2009;27(34):5838-5847.

49. Valabrega G, Montemurro F, Aglietta M. Trastuzumab: mechanism of action, resistance and future perspectives in HER2-overexpressing breast cancer. Ann Oncol. 2007;18(6):977-984.

50. Wolff AC, Hammond ME, Hicks DG, et al. Recommendations for human epidermal growth factor receptor 2 testing in breast cancer: American Society of Clinical Oncology/College of American Pathologists clinical practice guideline update. Arch Pathol Lab Med. 2014;138(2):241-256

51. Early Breast Cancer Trialists' Collaborative Group, Peto R, Davies C, et al. Comparisons between different polychemotherapy regimens for early breast cancer: meta-analyses of long-term outcome among 100,000 women in 123 randomised trials. Lancet. 2012;379(9814): 432-444.

52. Paik S. Development and clinical utility of a 21-gene recurrence score prognostic assay in patients with early breast cancer treated with tamoxifen. Oncologist. 2007;12(6):631-635.

53. Paik S, Shak S, Tang G, et al. A multigene assay to predict recurrence of tamoxifen-treated, node-negative breast cancer. $N$ Engl $\mathrm{J} \mathrm{Med}$. 2004;351(27):2817-2826.

54. Paik S, Tang G, Shak S, et al. Gene expression and benefit of chemotherapy in women with node-negative, estrogen receptor-positive breast cancer. J Clin Oncol. 2006;24(23):3726-3734.

55. Albain KS, Barlow WE, Shak S, et al. Prognostic and predictive value of the 21-gene recurrence score assay in postmenopausal women with node-positive, oestrogen-receptor-positive breast cancer on chemotherapy: a retrospective analysis of a randomised trial. Lancet Oncol. 2010;11(1):55-65.

56. Dowsett M, Cuzick J, Wale C, et al. Prediction of risk of distant recurrence using the 21-gene recurrence score in node-negative and node-positive postmenopausal patients with breast cancer treated with anastrozole or tamoxifen: a TransATAC study. J Clin Oncol. 2010;28(11):1829-1834. 
57. Mamounas EP, Tang G, Fisher B, et al. Association between the 21-gene recurrence score assay and risk of locoregional recurrence in nodenegative, estrogen receptor-positive breast cancer: results from NSABP B-14 and NSABP B-20. J Clin Oncol. 2010;28(10):1677-1683.

58. Tang G, Shak S, Paik S, et al. Comparison of the prognostic and predictive utilities of the 21-gene Recurrence Score assay and Adjuvant! for women with node-negative, ER-positive breast cancer: results from NSABP B-14 and NSABP B-20. Breast Cancer Res Treat. 2011;127(1): $133-142$.

59. Sparano JA, Gray RJ, Makower DF, et al. Prospective validation of a 21-gene expression assay in breast cancer. NEngl J Med. 2015;373(21): 2005-2014.

60. Hornberger J, Alvarado MD, Rebecca C, Gutierrez HR, Yu TM, Gradishar WJ. Clinical validity/utility, change in practice patterns, and economic implications of risk stratifiers to predict outcomes for early-stage breast cancer: a systematic review. J Natl Cancer Inst. 2012;104(14):1068-1079.

61. National Comprehensive Cancer Network Clinical Practice Guidelines in Oncology: Breast Cancer, version 3; 2015.

62. Harris L, Fritsche H, Mennel R, et al. American Society of Clinical Oncology 2007 update of recommendations for the use of tumor markers in breast cancer. J Clin Oncol. 2007;25(33):5287-5312.

63. US Food and Drug Administration. Draft Guidance for Industry, Clinical Laboratories, and Staff: Framework for Regulatory Oversight of Laboratory Developed Tests (LDTs). US Food and Drug Administration; 2014. Available from: http://www.fda.gov/downloads/medicaldevices/ deviceregulationandguidance/guidancedocuments/ucm416685.pdf. Accessed January 8, 2016.

64. US Food and Drug Administration. Draft Guidance for Industry, Clinical Laboratories, and Staff: In Vitro Diagnostic Multivariate Index Assays. US Food and Drug Administration; 2007. Available from: http://www. fda.gov/downloads/MedicalDevices/.../ucm071455.pdf. Accessed January 8, 2016.

65. van’t Veer LJ, Dai H, van de Vijver MJ, et al. Gene expression profiling predicts clinical outcome of breast cancer. Nature. 2002;415(6871): $530-536$.

66. van de Vijver MJ, He YD, van't Veer LJ, et al. A gene-expression signature as a predictor of survival in breast cancer. $N$ Engl J Med. 2002;347(25):1999-2009.

67. Buyse M, Loi S, van't Veer L, et al. Validation and clinical utility of a 70-gene prognostic signature for women with node-negative breast cancer. J Natl Cancer Inst. 2006;98(17):1183-1192.

68. Bueno-de-Mesquita JM, van Harten WH, Retel VP, et al. Use of 70-gene signature to predict prognosis of patients with node-negative breast cancer: a prospective community-based feasibility study (RASTER). Lancet Oncol. 2007;8(12):1079-1087.
69. Drukker CA, Bueno-de-Mesquita JM, Retel VP, et al. A prospective evaluation of a breast cancer prognosis signature in the observational RASTER study. Int J Cancer. 2013;133(4):929-936.

70. Mook S, Schmidt MK, Viale G, et al. The 70-gene prognosis-signature predicts disease outcome in breast cancer patients with 1-3 positive lymph nodes in an independent validation study. Breast Cancer Res Treat. 2009;116(2):295-302.

71. Parker JS, Mullins M, Cheang MC, et al. Supervised risk predictor of breast cancer based on intrinsic subtypes. J Clin Oncol. 2009;27(8): $1160-1167$.

72. Nielsen TO, Parker JS, Leung S, et al. A comparison of PAM50 intrinsic subtyping with immunohistochemistry and clinical prognostic factors in tamoxifen-treated estrogen receptor-positive breast cancer. Clin Cancer Res. 2010;16(21):5222-5232.

73. Dowsett M, Sestak I, Lopez-Knowles E, et al. Comparison of PAM50 risk of recurrence score with oncotype DX and IHC4 for predicting risk of distant recurrence after endocrine therapy. J Clin Oncol. 2013;31(22): 2783-2790.

74. Filipits M, Nielsen TO, Rudas M, et al. The PAM50 risk-of-recurrence score predicts risk for late distant recurrence after endocrine therapy in postmenopausal women with endocrine-responsive early breast cancer. Clin Cancer Res. 2014;20(5):1298-1305.

75. Gnant M, Filipits M, Greil R, et al. Predicting distant recurrence in receptor-positive breast cancer patients with limited clinicopathological risk: using the PAM50 Risk of Recurrence score in 1478 postmenopausal patients of the ABCSG- 8 trial treated with adjuvant endocrine therapy alone. Ann Oncol. 2014;25(2):339-345.

76. Sestak I, Cuzick J, Dowsett M, et al. Prediction of late distant recurrence after 5 years of endocrine treatment: a combined analysis of patients from the Austrian breast and colorectal cancer study group 8 and arimidex, tamoxifen alone or in combination randomized trials using the PAM50 risk of recurrence score. J Clin Oncol. 2015;33(8): 916-922.

77. Nielsen T, Wallden B, Schaper C, et al. Analytical validation of the PAM50-based Prosigna Breast Cancer Prognostic Gene Signature Assay and nCounter Analysis System using formalin-fixed paraffin-embedded breast tumor specimens. BMC Cancer. 2014;14(1):177.

78. US Food and Drug Administration. [webpage on the Internet]. List of cleared or approved companion diagnostic devices (in vitro and imaging tools). Available from: http:/www.fda.gov/MedicalDevices/ ProductsandMedicalProcedures/InVitroDiagnostics/ucm301431.htm. Accessed December 22, 2015.
Pharmacogenomics and Personalized Medicine

\section{Publish your work in this journal}

Pharmacogenomics and Personalized Medicine is an international, peerreviewed, open access journal characterizing the influence of genotype on pharmacology leading to the development of personalized treatment programs and individualized drug selection for improved safety, efficacy and sustainability. This journal is indexed on the American Chemical

\section{Dovepress}

Society's Chemical Abstracts Service (CAS). The manuscript management system is completely online and includes a very quick and fair peer-review system, which is all easy to use. Visit http://www.dovepress. com/testimonials.php to read real quotes from published authors. 